\title{
Effectiveness of obesity interventions among South Korean children and adolescents and importance of the type of intervention component: a meta-analysis
}

\author{
Siyoung Choe, $\mathrm{MPH}^{1}$, Jaesin Sa, $\mathrm{PhD}^{2}$, Jean-Philippe Chaput, $\mathrm{PhD}^{3}$, Deokjin Kim, $\mathrm{PhD}^{4}$
}

${ }^{1}$ Department of Kinesiology and Health, Miami University, Oxford, OH, USA; ${ }^{2}$ Department of Health and Human Performance, University of Tennessee at Chattanooga, Chattanooga, TN, USA; ${ }^{3}$ Healthy Active Living and Obesity Research Group, Children's Hospital of Eastern Ontario Research Institute, Ottawa, ON, Canada;

${ }^{4}$ Department of Sport \& Health Care, Namseoul University, Cheonan, Korea

Background: Various interventions have been tested to prevent or treat childhood obesity in South Korea. However, the overall effect of those interventions is unclear, as very few reviews and meta-analyses were specific to Korean children and adolescents.

Purpose: We aimed to examine the overall effect of obesity interventions among Korean children and adolescents, while also examining differences by sex, age group, baseline weight category, intervention duration, number of intervention components, and type of intervention components.

Methods: A meta-analysis was conducted for all intervention studies sampling Korean children and adolescents, with at least one control group and one month of follow-up, published between January 2000 and August 2020. Cohen d was calculated as an effect size for treatment effect, using the standardized difference between intervention group's body mass index (BMI) change and control group's BMI change.

Results: The final sample included 19 intervention studies with 2,140 Korean children (mean age, 12.2 years). Overall, interventions were strongly favored over their controls $(d=$ $1.61 ; 95 \%$ confidence interval [CI], 1.12-2.09). The subgroup analysis showed that interventions with at least one physical activity component $(d=2.43$; $95 \% \mathrm{CI}, 1.63-3.24)$ were significantly better than those that did not include physical activity $(d=0.02 ; 95 \%$ CI, -0.26 to 0.31$)$.

Conclusion: Type of intervention component appeared im. portant, though no differential association was observed by sex, age, baseline weight category, intervention duration, and number of intervention components. Korean and non-Korean interventions may be substantively different. Additional studies are needed to understand why and how Korean interventions differ from non-Korean interventions.

Key words: Weight management interventions, Body mass index, Child, Adolescent

\section{Key message}

Question: What is the overall effect of obesity interventions among Korean children and what affects their effectiveness?

Finding: Interventions were strongly favored over controls. Interventions including at least one physical activity component were significantly better than those that did not. Sex, age, baseline weight category, intervention duration, and the number of intervention components were not significant.

Meaning: Future obesity interventions for Korean children must seek to include physical activity components.

\section{Introduction}

The childhood obesity epidemic has become a major public health issue internationally. ${ }^{1)}$ According to the World Health Organization, more than 340 million children and adolescents in the world had overweight or obesity in 2016, and the global prevalence of childhood obesity has sharply increased in recent years. ${ }^{2)}$ The childhood obesity epidemic is of major concern not only to Western countries but also to Asian countries. ${ }^{3)}$ Research has shown that the prevalence of childhood obesity was highest in Asia among developing countries. ${ }^{4)}$ Among Asian countries, the prevalence of childhood obesity in South Korea (hereafter 'Korea') is high ${ }^{5)}$ and a sharp rise in the obesity prevalence among Korean children and adolescents (hereafter 'children') was noted over the last 3 decades. ${ }^{6}$ Nationally representative data from the Korean National School Health Examination survey using selfreported height and weight of survey participants showed that the prevalence of childhood obesity in Korea increased from 8.7\% in 2007 to $15.0 \%$ in 2017.7$)$

Previous studies found that childhood obesity in Korea is associated with body dissatisfaction, ${ }^{6}$ lower self-esteem, depression, ${ }^{8)}$ asthma, ${ }^{9)}$ lower health-related quality of life, ${ }^{10)}$ insulin resistance, and hypertension. ${ }^{6}$ In addition to the childhood obesity-related health problems, children with overweight or obesity may be at a

Corresponding author: Deokjin Kim, PhD, Department of Sport \& Health Care, Namseoul University, Daehak-ro, Seonghwan-eup, Seobuk-gu, Cheonan 31020, Korea 凶Email: kdj275@nsu.ac.kr, https://orcid.org/0000-0003-2388-4913

Received: 7 April, 2021, Revised: 13 October, 2021, Accepted: 14 October, 2021

This is an open-access article distributed under the terms of the Creative Commons Attribution Non-Commercial License (http://creativecommons.org/licenses/bync/4.0/) which permits unrestricted non-commercial use, distribution, and reproduction in any medium, provided the original work is properly cited.

Copyright (c) 2022 by The Korean Pediatric Society 

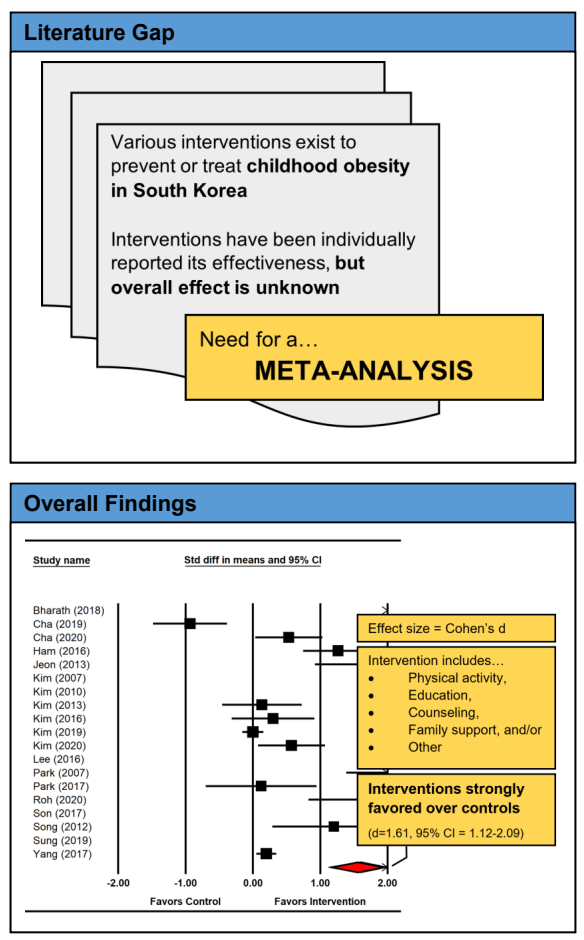

Graphic abstract

greater risk of excess weight during adulthood. ${ }^{11)}$ This could lead to an increased risk of cardiovascular disease ${ }^{12)}$ and several malig. nancies such as colorectal cancer ${ }^{13)}$ over an extended period of time.

Although various interventions have been performed to prevent or treat childhood obesity in Korea, the overall effect of those interventions remains unclear. ${ }^{14)}$ Pediatricians in Korean generally recommend multidisciplinary behavioral interventions for the treatment of childhood obesity, ${ }^{14)}$ but evidence for such a recommendation is based on studies ${ }^{15-18)}$ conducted outside of Korea. In Western countries, many meta-analyses have been performed to investigate the effect of obesity interventions among adults ${ }^{19-24)}$ and children. ${ }^{15-18,25-27)}$ Very few meta-analyses ${ }^{19,28)}$ have been conducted in Korea. Furthermore, these studies included both children and adults in the meta-analyses. None of the metaanalyses solely focused on obesity interventions for Korean children. Although a recent meta-analysis ${ }^{29)}$ was performed to analyze effects of obesity interventions conducted between 2000 and 2010, many of the studies included in this meta-analysis were outdated and did not directly measure individuals' adiposity. Moreover, the meta-analysis had an insufficient number of studies on adolescents (i.e., only 3 studies included middle and highschool students), was missing quality assessment of included studies, and was missing subgroup analyses by age and sex.

Given the lack of recent studies, the purpose of this meta-analysis was to address this gap in the literature by quantitatively investigating the effect of obesity interventions for Korean children and adolescents. We also explored possible sources of heterogeneity among interventions, by documenting how individual (e.g., sex, age group, and baseline weight category) and intervention
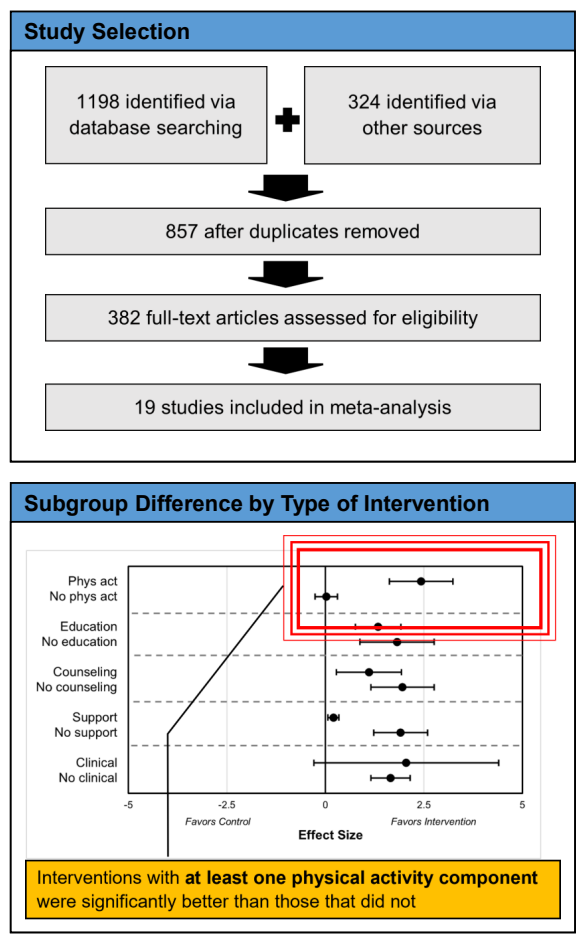

characteristics (e.g., intervention duration, number of intervention components, and type of intervention components) affect interventions' effectiveness. Surgical and pharmacological treatments are beyond the scope of this meta-analysis, as we have focused on interventions geared toward lifestyle and behavioral modification.

\section{Methods}

\section{Search strategy}

Systematic literature searches involved electronic keyword search of the following 8 databases, via EBSCOhost: MEDLINE; Science Citation Index; Science Direct; CINAHL Plus; Social Sciences Citation Index; APA PsychInfo; SPORTSDiscus; and Educational Resource Information Center. Search terms were a combination of obes", overweight, fat, BMI, body, mass, weight*, Korea", child*, adolescen*, youth, intervention, and trial. Manual search was conducted for a comprehensive coverage of the literature, by reviewing all references in eligible studies. A flowchart of study search and selection is shown in Fig. 1, using the template provided by the PRISMA (Preferred Reporting Items for Systematic Reviews and Meta-Analyses) guideline. ${ }^{30}$

\section{Inclusion and exclusion criteria}

Studies were selected if they met all of the following inclusion criteria: (1) peer-reviewed primary studies with full-text availability, published in English or Korean language between January 2000 and August 2020; (2) sampling Korean children and adolescents, aged 2-21 years; (3) intervention studies with at least one 
control group and one month of follow-up; and (4) studies with data on mean body mass index (BMI) change between baseline and follow-up.

Studies were excluded if they met any of the following exclusion criteria: (1) studies with cross-sectional design, less than one month of follow-up, or no control group; (2) studies with data or cohort overlapping with another study; (3) studies with unclear demographics information, to assure that findings were applicable to the Korean population; and (4) studies that primarily sampled ungeneralizable populations (e.g., foreign individuals with Korean heritage, immigrants and visitors in Korea, and individuals who are incarcerated or hospitalized long term). We further excluded studies that reported only body mass, BMI percentile, $\mathrm{BMI} z$ score, or percent adiposity change, after sensitivity analysis found a significant difference between studies that directly reported BMI change and those that used estimated BMI change. We also excluded one study that used nonstandard formula to calculate BMI. As shown in Fig. 1, of the 382 identified articles that seemed to provide relevant information, 19 fulfilled the inclusion criteria and were thus included in this paper. The majority of included studies were randomized controlled trials, though three ${ }^{31-33)}$ were quasi-experimental by design, and one had unclear randomization protocol. ${ }^{34}$

\section{Data extraction}

Studies were reviewed by investigators to extract and sum. marize relevant data. Data extracted from each study included: (1) general information, such as author names and year of publication; (2) study characteristics, such as study design, sample size, and length of follow-up; (3) participant characteristics, such as age and sex; and (4) estimates of BMI change and 95\% confidence

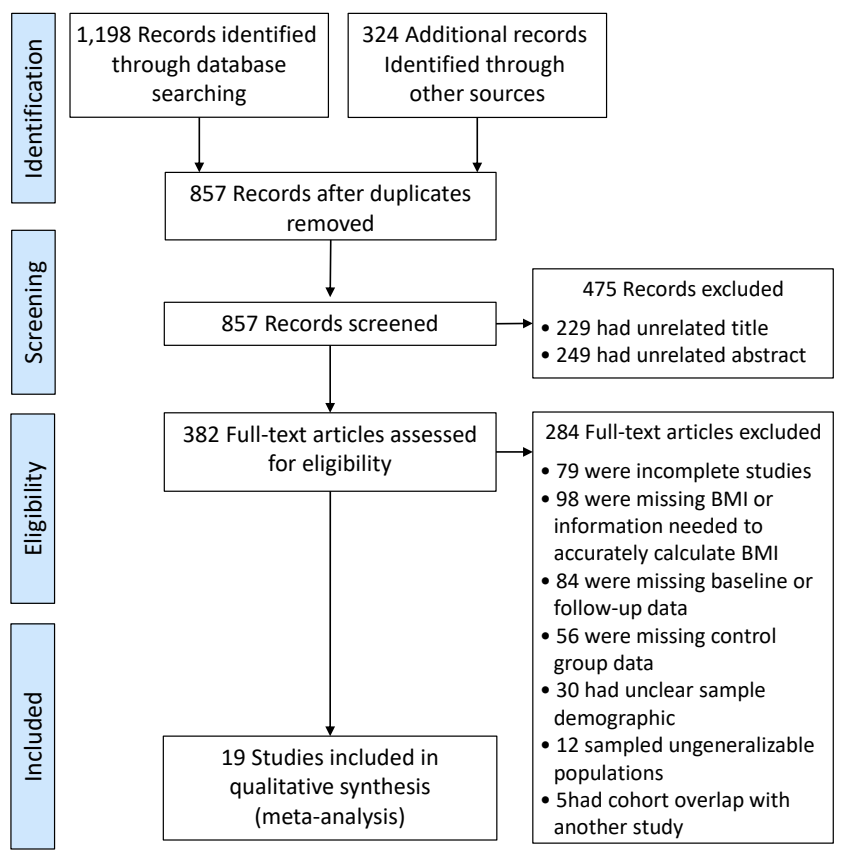

Fig. 1. PRISMA (Preferred Reporting Items for Systematic Reviews and Meta-Analyses) flowchart. intervals (CIs), or numbers needed to calculate BMI change and 95\% CI. When BMI values were not directly reported, they were manually calculated using the formula: $\mathrm{BMI}=$ weight (in $\mathrm{kg}$ )/ height $^{2}$ (in meters).

When there were multiple studies published from one research cohort, data were extracted from the study that reported more details and had more information for quality assessment. When there were more than one follow-up data point, the data point that immediately followed the end of the intervention was used.

Information on participants' sex, age group, and baseline weight category were extracted for subgroup analysis. The number of intervention components, type of intervention components included, and intervention duration were also extracted for subgroup analysis. Sex (boy vs. girl), age group (children vs. adolescent), and baseline weight category (under/normal weight vs. overweight/obesity) were each recoded as dichotomous variables. Baseline weight category was taken directly from each study, all of which were determined by BMI percentile or clinical diagnosis. Type of intervention component included (physical activity, group education, counseling, family support, and other component) was recoded as 5 separate binary (yes vs. no) variables. The number of intervention components (1 vs. 2 vs. 3 vs. 5) and intervention duration (1-2 months vs. $3-4$ months vs. 5 or more months) were respectively recoded as 4-category and 3-category variables.

When recoding participants' age, definitions provided by the American Academy of Pediatrics were adapted to differentiate children and adolescents, such that children were individuals between ages 2-12 (typically attending pre- or elementary school), and adolescents were non-adults aged 13 or older (typically attending middle- or high school). ${ }^{35}$ )

\section{Bias assessment}

Risk of bias for each study was assessed using the Cochrane Collaboration tool, ${ }^{36}$ ) which guided interpretation of the study results. Criteria for assessment included: sequence, allocation, blinding-person, blinding-measurement, completion, and selection. Studies showing clear bias in any one of these criteria were marked with a minus (-) sign, while studies with practice that minimize bias were marked with a plus $(+)$ sign. Studies were then assessed for overall quality, where studies with poor overall quality were excluded from final analysis. Studies included in final analysis were also tested for publication bias by plotting and visually inspecting precision funnel plot, followed by Begg test. Duval and Tweedie trim and fill were attempted for imputation and correcting publication bias.

\section{Data analysis}

Methods used for data analysis were modeled after an earlier meta-analysis. ${ }^{25)}$ Each study was used as a unit of analysis for the overall treatment effect. Cohen $d$ was calculated as an effect size for treatment effect in each controlled study. Cohen $d$ was computed by dividing mean differences of intervention and control groups by the average baseline standard deviation of the 2 groups. 
37) Positive effect sizes mean favorable changes in comparison with the control group. Random effects model was used over fixed effects model due to relatively high degree of heterogeneity among studies. Heterogeneity was determined by conducting Cochran $Q$ tests and examining $I^{2}$ statistics, where $I^{2}$ values of 25,50 , and 75 correspond to small, moderate, and large heterogeneity. ${ }^{38)}$

Subgroup analysis was performed among studies with available data to identify the potential source of heterogeneity. Sex, age group, baseline weight category, the number of intervention components, intervention duration, and type of intervention components were included as independent moderating variables for subgroup analysis. Each subgroup analysis included a test for between-groups heterogeneity. ${ }^{39)}$ All analyses were performed using Comprehensive Meta-Analysis software version 2.0 (Biostat Inc., Englewood, NJ, USA). ${ }^{40)}$

\section{Results}

\section{Descriptive statistics}

This meta-analysis included 19 intervention studies, involving
38 study arms (19 treatment arms and 19 control arms), with 2,140 Korean children and adolescents. The number of total study participants ranged from 20 to 768 (mean, 113 participants). The mean age of participants ranged from 4.5 to 16.0 years (mean, 12.2 years) at baseline, and the mean intervention duration ranged from 5 weeks to 1 year (mean, 15.4 weeks).

Of the 19 studies, 2, 4, and 13 studies sampled boys only, girls only, and both boys and girls, respectively. Of these 19, 14 sampled only participants with overweight or obesity at baseline, while 5 used participants regardless of their baseline body weight. Nine studies sampled only children, 8 sampled only adolescents, and 2 sampled both children and adolescents.

Ten studies used one-component interventions, 4 two-component interventions, 4 three-component interventions, and only 1 study used five components (Yang et al.'s study). ${ }^{33)}$ Regardless of the number of intervention components, there were a total of 14 studies with at least one physical activity component as part of its intervention, and 5 studies that did not have a physical activity component. Nine studies had at least one group education component, while 10 did not have an education component. Five had a counseling component, while 14 were missing a counseling component. There were also 2 studies with, and 17 without, a

Table 1. Characteristics of included studies

\begin{tabular}{|c|c|c|c|c|c|c|}
\hline Study & Intervention description & $\begin{array}{l}\text { Duration } \\
\text { (wk) }\end{array}$ & Sample description & $\begin{array}{c}\% \\
\text { Boys }\end{array}$ & $\begin{array}{l}\text { Mean age } \\
(y r)\end{array}$ & $\begin{array}{l}\text { Baseline } \\
\text { weight }\end{array}$ \\
\hline $\begin{array}{l}\text { Bharath et al. } \\
(2018)^{48)}\end{array}$ & Combined exercise training (CRAE) & 12 & Adolescents (Tanner stage III-IV) & 0.0 & 14.7 & Ow/Ob \\
\hline Cha et al. $(2019)^{49)}$ & $\begin{array}{l}\text { Auricular acupressure on points: shen men, } \\
\text { stomach, spleen, hunge, endocrine }\end{array}$ & 8 & Adolescents (high school) & 55.2 & 16.0 & Ow/Ob \\
\hline Cha et al. $(2020)^{50)}$ & $\begin{array}{l}\text { Auricular acupressure on points: shen men, } \\
\text { stomach, spleen, hunge, endocrine }\end{array}$ & 8 & Children (elementary school) & - & 10.4 & Ow/Ob \\
\hline Ham et al. $(2016)^{11)}$ & $\begin{array}{l}\text { Skipping rope exercise+TTM-based multiple } \\
\text { exercise counseling+booster counseling }\end{array}$ & 26 & Children (elementary school) & 56.0 & 10.6 & $\mathrm{Ow} / \mathrm{Ob}$ \\
\hline Jeon et al. $(2013)^{51)}$ & $\begin{array}{l}\text { Combination exercise (walking+rubber band } \\
\text { exercise) }\end{array}$ & 12 & $\begin{array}{l}\text { Children (overweight, no CVD, no } \\
\text { exercise) }\end{array}$ & - & - & $\mathrm{Ow} / \mathrm{Ob}$ \\
\hline Kim et al. $(2007)^{52)}$ & PE class+supervised exercise session & 6 & Adolescents (middle school) & 100.0 & 17.0 & $\mathrm{Ow} / \mathrm{Ob}$ \\
\hline Kim et al. $(2010)^{53)}$ & $\begin{array}{l}\text { Physical activity+nutrition education+chromium } \\
\text { supplementation }\end{array}$ & 6 & Children (overweight) & 46.8 & 10.8 & Ow/Ob \\
\hline Kim et al. $(2013)^{31)}$ & Health coaching+emotional self-regulation training & 8 & Children & 52.2 & 9.0 & $\mathrm{Ow} / \mathrm{Ob}$ \\
\hline Kim et al. (2016) ${ }^{54)}$ & $\begin{array}{l}\text { Exercise+nutrition education+parent involvement } \\
\text { (newsletter \& text message) }\end{array}$ & 5 & $\begin{array}{l}\text { Children (with overweight or } \\
\text { obesity) }\end{array}$ & 57.2 & 9.7 & Ow/Ob \\
\hline Kim et al. (2019) & Dietary education (NASA MX) & 10 & Children (daycare \& kindergarten) & 56.4 & 4.5 & Mix \\
\hline Kim et al. $(2020)^{56)}$ & $\begin{array}{l}\text { Customized nutritional intervention+physical } \\
\text { activity }\end{array}$ & 16 & $\begin{array}{l}\text { Children \& adolescents (with } \\
\text { obesity) }\end{array}$ & 61.2 & 12.4 & Ow/Ob \\
\hline Lee et al. (2016) & $\begin{array}{l}\text { Intensive physical activity \& nutrition program } \\
\text { (education+practice+counseling) }\end{array}$ & 26 & Children (elementary school) & 55.4 & 11.0 & Mix \\
\hline Park et al. $(2007)^{57)}$ & Walking+lifestyle education & 12 & Adolescents (with obesity) & 0.0 & 14.2 & $\mathrm{Ow} / \mathrm{Ob}$ \\
\hline Park et al. $(2017)^{34)}$ & PE class participation & 8 & Children (elementary school) & 50.0 & 12.5 & Mix \\
\hline Roh et al. $(2020)^{58)}$ & TKD exercise & 16 & $\begin{array}{l}\text { Adolescents (with overweight or } \\
\text { obesity) }\end{array}$ & 70.0 & 12.6 & $\mathrm{Ow} / \mathrm{Ob}$ \\
\hline Son et al. $(2017)^{59)}$ & Combined exercise training (CRAE) & 12 & Adolescents (Tanner II-III stage) & 0.0 & 15.0 & $\mathrm{Ow} / \mathrm{Ob}$ \\
\hline Song et al. $(2012)^{60)}$ & Aerobic exercise & 12 & Adolescents (with overweight or obesity) & 100.0 & 12.7 & Ow/Ob \\
\hline Sung et al. $(2019)^{61)}$ & Jump rope exercise & 12 & Adolescents & 0.0 & 15.0 & Mix \\
\hline Yang et al. $(2017)^{33)}$ & $\begin{array}{l}\text { Health education+encouragement cues+per- } \\
\text { sonalized guidance+family involvement+ summer } \\
\text { program }\end{array}$ & 52 & $\begin{array}{l}\text { Children \& adolescents (elementary \& } \\
\text { middle school) }\end{array}$ & 75.9 & 10.9 & Mix \\
\hline
\end{tabular}

TTM, transtheoretical model; PE, physical education; TKD, taekwondo; Ow/Ob, overweight or obesity. 
family support component. Three had other atypical components (i.e., 1 chemical supplementation and 2 acupressure treatment), while 16 did not have any of those components. Characteristics of included studies are summarized in Table 1.

\section{Bias assessment}

Quality assessment is summarized in Table 2. Studies generally showed unclear risk of bias under each category. We found a sign of publication bias among included studies, based on asymme- trical distribution of the precision funnel plot (see Fig. 2) and Begg test $(1$-tailed $P<0.01)$. However, the point estimate did not notably change after inclusion of imputed studies using Duval and Tweedie trim and fill.

\section{Effect of interventions on BMI}

Overall, a significant treatment effect was observed. Combined effect size was $1.61\left(95 \% \mathrm{CI}, 1.12-2.09 ; I^{2}=94.8 ; P<0.001\right)$ in the random-effects model, indicating a significant $\mathrm{BMI}$ reduction

Table 2. Summary of study quality and risk of bias

\begin{tabular}{|c|c|c|c|c|c|c|c|c|}
\hline Study & Sequence & Allocation & Blinding-person & Blinding-measure & Incomplete & Selection & Other & Overall \\
\hline Bharath et al. (2018) ${ }^{48)}$ & $?$ & $?$ & $?$ & $?$ & $?$ & $?$ & $?$ & $?$ \\
\hline Cha et al. $(2019)^{49)}$ & + & + & $?$ & $?$ & $?$ & + & $?$ & + \\
\hline Cha et al. $(2020)^{50)}$ & + & + & $?$ & $?$ & + & + & $?$ & + \\
\hline Ham et al. (2016) ${ }^{11)}$ & $?$ & $?$ & $?$ & $?$ & $?$ & $?$ & - & $?$ \\
\hline Jeon et al. $(2013)^{51)}$ & $?$ & $?$ & $?$ & $?$ & $?$ & $?$ & - & $?$ \\
\hline Kim et al. $(2007)^{52)}$ & + & + & $?$ & $?$ & $?$ & $?$ & $?$ & + \\
\hline Kim et al. $(2010)^{53)}$ & + & $?$ & + & + & $?$ & + & $?$ & + \\
\hline Kim et al. $(2013)^{31)}$ & $?$ & $?$ & $?$ & $?$ & $?$ & $?$ & $?$ & $?$ \\
\hline Kim et al. $(2016)^{54)}$ & $?$ & $?$ & $?$ & $?$ & $?$ & $?$ & - & $?$ \\
\hline Kim et al. $(2019)^{55)}$ & $?$ & $?$ & $?$ & $?$ & $?$ & $?$ & $?$ & $?$ \\
\hline Kim et al. $(2020)^{56)}$ & $?$ & $?$ & $?$ & $?$ & $?$ & $?$ & $?$ & $?$ \\
\hline Lee et al. $(2016)^{32)}$ & + & + & + & + & + & - & $?$ & + \\
\hline Park et al. $(2007)^{57)}$ & $?$ & $?$ & $?$ & $?$ & $?$ & $?$ & $?$ & $?$ \\
\hline Park et al. $(2017)^{34)}$ & $?$ & $?$ & $?$ & $?$ & $?$ & $?$ & $?$ & $?$ \\
\hline Roh et al. $(2020)^{58)}$ & $?$ & $?$ & $?$ & $?$ & $?$ & $?$ & $?$ & $?$ \\
\hline Son et al. $(2017)^{59)}$ & $?$ & $?$ & $?$ & $?$ & $?$ & $?$ & $?$ & $?$ \\
\hline Song et al. $(2012)^{60)}$ & $?$ & $?$ & $?$ & $?$ & $?$ & $?$ & $?$ & $?$ \\
\hline Sung et al. $(2019)^{61)}$ & $?$ & $?$ & $?$ & $?$ & $?$ & $?$ & $?$ & $?$ \\
\hline Yang et al. $(2017)^{33)}$ & $?$ & - & - & $?$ & + & $?$ & $?$ & $?$ \\
\hline
\end{tabular}

+, low risk; -, high risk; ?, unclear risk.

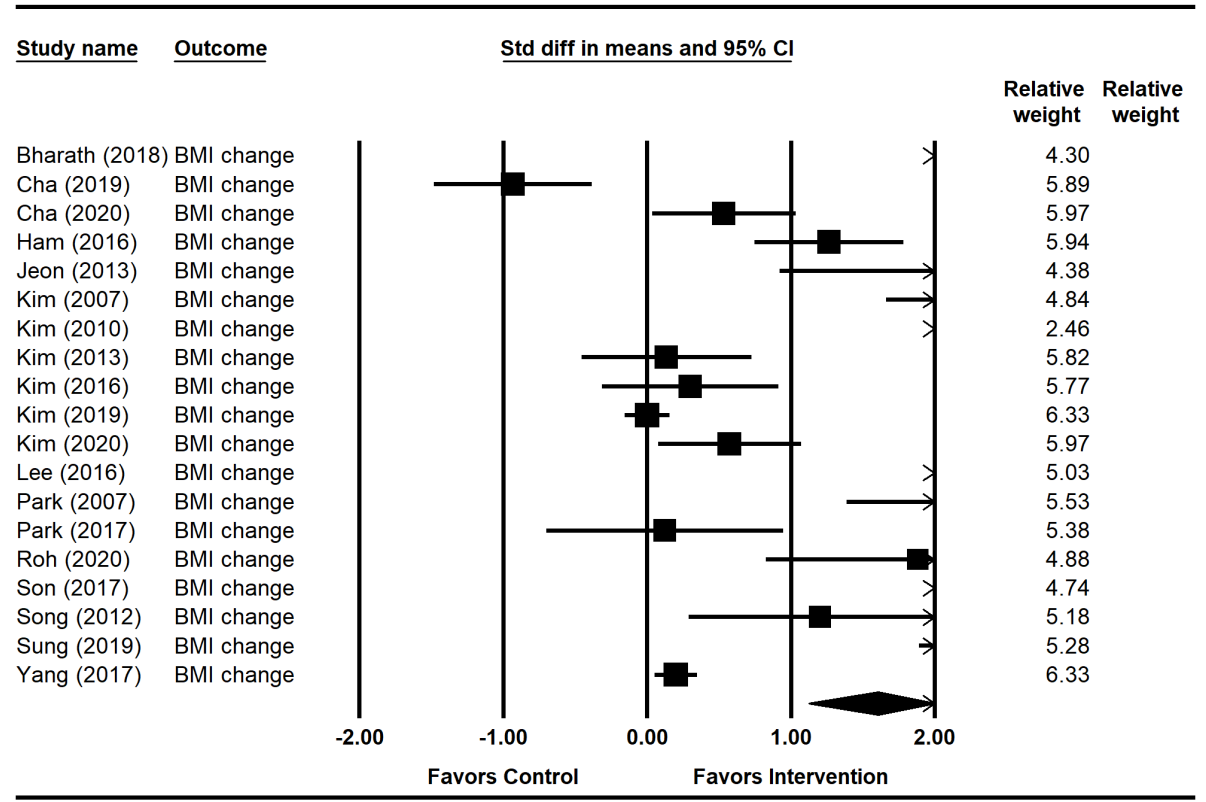

Fig. 2. Funnel plot of precision by standard difference in means for observed and imputed studies. Circles, individual studies; blank diamond, overall effect without imputation; filled diamond, overall effect after imputation. 
in the intervention-treated group compared to the control group. BMI change ranged from -3.00 to 0.79 for the intervention group (mean, -0.80), and -2.00 to 1.08 for the control group (mean, 0.09). Summary of intervention components and effect size data are found in Table 3 and Fig. 3.

\section{Subgroup analysis}

1) Comparison by participants' sex, age group, and baseline weight category

Significant treatment effect was observed in boys and girls among 6 studies reporting sex-specific data. In both sexes, significant
BMI reduction was observed in intervention groups compared to their controls. Among 6 studies with sex-specific data, 2 reported data on boys, with an effect size of 1.94 (95\% CI, 0.44-3.45; $\left.I^{2}=78.1 ; P=0.011\right)$. Four reported data on girls, with an effect size of 3.53 (95\% CI, 2.21 -4.86; $\left.I^{2}=86.4 ; P<0.001\right)$. Despite significant treatment effect, the magnitude of effect sizes did not significantly differ in boys and girls, and no between-groups heterogeneity was observed $(P=0.12)$.

Among 17 studies reporting age-group specific data, treatment effect was significant in both age groups (i.e., children and adolescents). Among these 17 studies, 9 reported data on children,

Table 3. Summary of intervention components and data

\begin{tabular}{|c|c|c|c|c|c|c|c|c|c|c|c|c|c|}
\hline \multirow[b]{2}{*}{ Study } & \multirow{2}{*}{$\begin{array}{c}\text { No. of } \\
\text { intervention } \\
\text { components }\end{array}$} & \multicolumn{5}{|c|}{ Included component } & \multirow{2}{*}{\multicolumn{2}{|c|}{$\Delta^{\mathrm{a})}$}} & \multirow[b]{2}{*}{ SD } & \multirow[b]{2}{*}{ No. } & \multirow{2}{*}{$\begin{array}{c}\text { Effect } \\
\text { size } \\
(\text { Cohen } d)^{\text {b) }}\end{array}$} & \multirow[b]{2}{*}{$95 \% \mathrm{Cl}$} & \multirow{2}{*}{$\begin{array}{c}P \\
\text { value }\end{array}$} \\
\hline & & $\begin{array}{c}\text { PA/ } \\
\text { exercise }\end{array}$ & Education & Counseling & $\begin{array}{l}\text { Family } \\
\text { support }\end{array}$ & Other & & & & & & & \\
\hline $\begin{array}{l}\text { Bharath et al. } \\
(2018)^{48)}\end{array}$ & 1 & Yes & No & No & No & No & $\begin{array}{l}\text { l: } \\
\text { C: }\end{array}$ & $\begin{array}{r}-3.000 \\
0.000\end{array}$ & $\begin{array}{l}0.680 \\
0.412\end{array}$ & $\begin{array}{l}20 \\
20\end{array}$ & 5.336 & $4.013-6.66$ & 0.000 \\
\hline $\begin{array}{l}\text { Cha et al. } \\
(2019)^{49)}\end{array}$ & 1 & No & No & No & No & Yes & $\begin{array}{l}\text { l: } \\
\text { C: }\end{array}$ & $\begin{array}{l}0.790 \\
0.140\end{array}$ & $\begin{array}{l}0.604 \\
0.795\end{array}$ & $\begin{array}{l}32 \\
26\end{array}$ & -0.934 & -1.479 to -0.390 & 0.001 \\
\hline $\begin{array}{l}\text { Cha et al. } \\
(2020)^{50)}\end{array}$ & 1 & No & No & No & No & Yes & $\begin{array}{l}\text { l: } \\
\text { C: }\end{array}$ & $\begin{array}{r}-0.210 \\
0.100\end{array}$ & $\begin{array}{l}0.691 \\
0.460\end{array}$ & $\begin{array}{l}31 \\
34\end{array}$ & 0.533 & $0.038-1.028$ & 0.035 \\
\hline $\begin{array}{l}\text { Ham et al. } \\
(2016)^{11)}\end{array}$ & 3 & Yes & No & Yes & No & No & $\begin{array}{l}\text { l: } \\
\text { C: }\end{array}$ & $\begin{array}{l}0.020 \\
0.770\end{array}$ & $\begin{array}{l}0.557 \\
0.653\end{array}$ & $\begin{array}{l}48 \\
27\end{array}$ & 1.265 & $0.752-1.778$ & 0.000 \\
\hline $\begin{array}{l}\text { Jeon et al. } \\
(2013)^{51)}\end{array}$ & 1 & Yes & No & No & No & No & $\begin{array}{l}\text { l: } \\
\text { C: }\end{array}$ & $\begin{array}{r}-2.270 \\
0.130\end{array}$ & $\begin{array}{l}1.230 \\
0.888\end{array}$ & $\begin{array}{l}8 \\
7\end{array}$ & 2.211 & $0.924-3.497$ & 0.001 \\
\hline $\begin{array}{l}\text { Kim et al. } \\
(2007)^{52)}\end{array}$ & 2 & Yes & Yes & No & No & No & $\begin{array}{l}\text { l: } \\
\text { C: }\end{array}$ & $\begin{array}{l}-1.000 \\
-0.300\end{array}$ & $\begin{array}{l}0.227 \\
0.286\end{array}$ & $\begin{array}{l}14 \\
12\end{array}$ & 2.737 & $1.666-3.809$ & 0.000 \\
\hline $\begin{array}{l}\text { Kim et al. } \\
(2010)^{53)}\end{array}$ & 3 & Yes & Yes & No & No & Yes & $\begin{array}{l}\text { l: } \\
\text { C: }\end{array}$ & $\begin{array}{l}-0.220 \\
-0.009\end{array}$ & $\begin{array}{l}0.020 \\
0.030\end{array}$ & $\begin{array}{l}12 \\
13\end{array}$ & 8.208 & $5.801-10.614$ & 0.000 \\
\hline $\begin{array}{l}\text { Kim et al. } \\
(2013)^{31)}\end{array}$ & 2 & No & Yes & Yes & No & No & $\begin{array}{l}\text { l: } \\
\text { C: }\end{array}$ & $\begin{array}{l}0.000 \\
0.100\end{array}$ & $\begin{array}{l}0.638 \\
0.807\end{array}$ & $\begin{array}{l}19 \\
27\end{array}$ & 0.135 & -0.453 to 0.722 & 0.653 \\
\hline $\begin{array}{l}\text { Kim et al. } \\
(2016)^{54)}\end{array}$ & 3 & Yes & Yes & No & Yes & No & $\begin{array}{l}\text { l: } \\
\text { C: }\end{array}$ & $\begin{array}{r}-0.240 \\
0.000\end{array}$ & $\begin{array}{l}0.796 \\
0.814\end{array}$ & $\begin{array}{l}23 \\
19\end{array}$ & 0.298 & -0.313 to 0.909 & 0.338 \\
\hline $\begin{array}{l}\text { Kim et al. } \\
(2019)^{55)}\end{array}$ & 1 & No & Yes & No & No & No & $\begin{array}{l}\text { l: } \\
\text { C: }\end{array}$ & $\begin{array}{l}-0.300 \\
-0.300\end{array}$ & $\begin{array}{l}0.111 \\
0.119\end{array}$ & $\begin{array}{l}339 \\
340\end{array}$ & 0.000 & -0.150 to 0.150 & 1.000 \\
\hline $\begin{array}{l}\text { Kim et al. } \\
(2020)^{56)}\end{array}$ & 2 & Yes & No & Yes & No & No & $\begin{array}{l}\text { l: } \\
\text { C: }\end{array}$ & $\begin{array}{r}-0.700 \\
0.000\end{array}$ & $\begin{array}{l}0.885 \\
1.500\end{array}$ & $\begin{array}{l}34 \\
32\end{array}$ & 0.573 & $0.080-1.065$ & 0.023 \\
\hline $\begin{array}{l}\text { Lee et al. } \\
(2016)^{32)}\end{array}$ & 3 & Yes & Yes & Yes & No & No & $\begin{array}{l}\text { l: } \\
\text { C: }\end{array}$ & $\begin{array}{r}-1.120 \\
1.080\end{array}$ & $\begin{array}{l}0.375 \\
0.703\end{array}$ & $\begin{array}{l}23 \\
23\end{array}$ & 3.905 & $2.920-4.890$ & 0.000 \\
\hline $\begin{array}{l}\text { Park et al. } \\
(2007)^{57)}\end{array}$ & 2 & Yes & Yes & No & No & No & $\begin{array}{l}\text { l: } \\
\text { C: }\end{array}$ & $\begin{array}{l}-1.800 \\
-0.100\end{array}$ & $\begin{array}{l}0.890 \\
0.694\end{array}$ & $\begin{array}{l}22 \\
22\end{array}$ & 2.130 & $1.390-2.870$ & 0.000 \\
\hline $\begin{array}{l}\text { Park et al. } \\
(2017)^{34)}\end{array}$ & $1^{*}$ & Yes & Yes & No & No & No & $\begin{array}{l}\text { l: } \\
\text { C: }\end{array}$ & $\begin{array}{l}0.170 \\
0.410\end{array}$ & $\begin{array}{l}1.655 \\
2.172\end{array}$ & $\begin{array}{l}11 \\
12\end{array}$ & 0.124 & -0.695 to 0.942 & 0.768 \\
\hline $\begin{array}{l}\text { Roh et al. } \\
(2020)^{58)}\end{array}$ & 1 & Yes & No & No & No & No & $\begin{array}{l}\text { l: } \\
\text { C: }\end{array}$ & $\begin{array}{r}-1.320 \\
0.080\end{array}$ & $\begin{array}{l}0.836 \\
0.637\end{array}$ & $\begin{array}{l}10 \\
10\end{array}$ & 1.884 & $0.831-2.937$ & 0.000 \\
\hline $\begin{array}{l}\text { Son et al. } \\
(2017)^{59)}\end{array}$ & 1 & Yes & No & No & No & No & $\begin{array}{l}\text { l: } \\
\text { C: }\end{array}$ & $\begin{array}{l}-2.970 \\
-2.000\end{array}$ & $\begin{array}{l}0.221 \\
0.237\end{array}$ & $\begin{array}{l}20 \\
20\end{array}$ & 4.233 & $3.118-5.349$ & 0.000 \\
\hline $\begin{array}{l}\text { Song et al. } \\
(2012)^{60)}\end{array}$ & 1 & Yes & No & No & No & No & $\begin{array}{l}\text { l: } \\
\text { C: }\end{array}$ & $\begin{array}{l}-0.500 \\
-0.100\end{array}$ & $\begin{array}{l}0.348 \\
0.313\end{array}$ & $\begin{array}{l}12 \\
10\end{array}$ & 1.202 & $0.291-2.114$ & 0.010 \\
\hline $\begin{array}{l}\text { Sung et al. } \\
(2019)^{61)}\end{array}$ & 1 & Yes & No & No & No & No & $\begin{array}{l}\text { I: } \\
\text { C: }\end{array}$ & $\begin{array}{r}-1.000 \\
1.000\end{array}$ & $\begin{array}{l}0.806 \\
0.632\end{array}$ & $\begin{array}{l}20 \\
20\end{array}$ & 2.761 & $1.895-3.628$ & 0.000 \\
\hline $\begin{array}{l}\text { Yang et al. } \\
(2017)^{33)}\end{array}$ & 5 & No & Yes & Yes & Yes & No & $\begin{array}{l}\text { l: } \\
\text { C: }\end{array}$ & $\begin{array}{l}0.400 \\
0.700\end{array}$ & $\begin{array}{l}1.432 \\
1.565\end{array}$ & $\begin{array}{l}350 \\
418\end{array}$ & 0.199 & $0.057-0.342$ & 0.006 \\
\hline $\begin{array}{l}\text { Pooled } \\
\text { (random) }\end{array}$ & & & & & & & & & & & 1.605 & $1.124-2.086$ & 0.000 \\
\hline
\end{tabular}

PA, physical activity; SD, standard deviation; $\mathrm{Cl}$, confidence interval, I, intervention group; C, control group.

Negative Cohen d indicates unfavorable changes in the intervention group compared to the control group (i.e., weight increase from A to B or higher body mass index in the intervention group than in the control group at posttest). Interventions may include more than one component per type of intervention component. The number of yes under included components may not add up to the number under number of intervention components.

a) Mean difference=posttest-pretest. ${ }^{\text {b) }}$ Formula used is (mean change for intervention-mean change for control)/([SD at baseline for intervention and control]/2). 


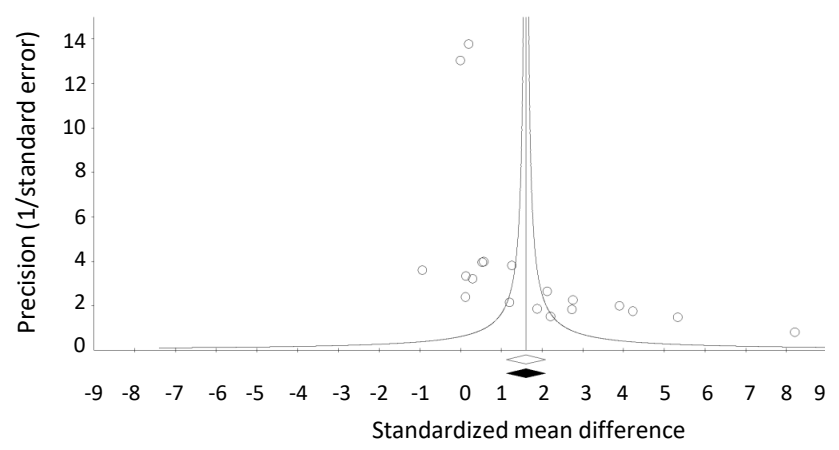

Fig. 3. Forest plot summarizing individual and pooled effect size. Squares, individual effect sizes; bars, 95\% confidence intervals; diamond, overall effect size (random effects model).

with an effect size of 1.39 (95\% CI, 0.63-2.15; $I^{2}=93.9 ; P<$ 0.001). Eight reported data on adolescents, with an effect size of 2.38 (95\% CI, 0.93-3.83; $\left.I^{2}=95.3 ; P=0.001\right)$. No significant difference was observed by participant's age group $(P=0.12)$.

Treatment effect was significant regardless of participants' baseline weight category, when examining all included studies. Fourteen studies that sampled only participants with overweight or obesity at baseline had an effect size of 1.88 (95\% CI, 1.102.66; $I^{2}=93.6 ; P<0.001$ ), while 5 studies that sampled participants across all weight categories had an effect size of 1.17 (95\% CI, 0.51-1.82; $P=95.8 ; P<0.001)$. However, no significant difference was observed between the 2 groups $(P=0.17)$ (Supplementary Figs. 1-4)

2) Comparison by number, duration, and type of intervention components

The number of intervention components was a significant moderator, showing significant treatment effect across all levels. Effect sizes were 1.63 (95\% CI, 0.73-2.54; $\left.I^{2}=95.3 ; P<0.001\right)$ for 10 one-component studies, 1.33 (95\% CI, $0.25-2.40 ; I^{2}=$ 90.0; $P=0.015$ ) for 4 two-component studies, 3.05 (95\% CI, 1.09-5.01; $\left.I^{2}=95.6 ; P=0.002\right)$ for 4 three-component studies, and 0.20 (95\% CI, $\left.0.06-0.34 ; I^{2}=0.00 ; P=0.006\right)$ for 1 fivecomponent study. Total between-groups heterogeneity was significant at $P<0.001$.

Intervention duration was significant across all levels, with effect sizes of 1.10 (95\% CI, 0.10-2.10; $I^{2}=92.7 ; P=0.032$; $\mathrm{n}=7$ ) for interventions lasting 1-2 months, 2.19 (95\% CI, 1.13$\left.3.26 ; I^{2}=95.9 ; P<0.001 ; \mathrm{n}=9\right)$ for $3-4$ months, and $1.71(95 \%$ CI, $0.12-3.30 ; I^{2}=97.1 ; P=0.035 ; \mathrm{n}=3$ ) for 5 or more months. However, total between-groups heterogeneity showed no significance $(P=0.340)$, and intervention duration was not a significant moderator.

When considering different types of intervention components, only 2 (i.e., physical activity component and family support component) out of 5 components were significant moderators (both $P<0.001$ for tests of between-groups heterogeneity). Studies with at least one physical activity component in its interventions showed significant treatment effect (effect size $=2.43 ; 95 \%$ CI, 1.63-3.24; $P=92.1 ; P<0.001 ; \mathrm{n}=14)$, while no significant effect was found in studies with no physical activity component (effect size $=0.02 ; 95 \% \mathrm{CI},-0.26$ to $0.31 ; P=80.3 ; P=0.868$; $\mathrm{n}=5$ ). Studies showed significant treatment effect whether or not they included a family support component. However, the magnitude of treatment effect was greater in studies without family support, such that effect sizes were 0.20 (95\% CI, 0.07$0.34 ; P=0.00 ; P=0.004 ; \mathrm{n}=2$ ) for studies with a family support component, and 1.91 (95\% CI, 1.23-2.59; $P=95.2 ; P<0.001$; $\mathrm{n}=17$ ) for studies without a family support component. None of the remaining 3 moderators (i.e., group education, counseling, and other component) showed significant between-groups heterogeneity (respective $P$ 's $=0.391,0.148$, and 0.745 ).

\section{Discussion}

Many interventions have been conducted to address childhood obesity in Korea. However, no reviews have been published that have compiled the scientific evidence about the effectiveness of childhood obesity interventions in Korea. To best of our knowledge, this is one of the first meta-analyses to examine the combined effect of obesity interventions in Korean children and adolescents. We also conducted subgroup analyses to explore possible source of heterogeneity. This review is important to help inform research, policy, and practice pertaining to childhood obesity interventions in Korea.

We found that, overall, intervention group children showed more favorable changes than control group children, as the 2 groups differed by 1.61 standard deviations. Our findings had the direction of effect that was consistent with earlier metaanalyses, ${ }^{25,29)}$ but the magnitude of effect was notably greater than the one found in U.S. minority children (median effect size $=0.16) .{ }^{25)}$ Such difference may be attributed to the limitations of studies included in our analysis. We found evidence of publication bias, suggesting that obesity interventions in Korean children may have been unpublished if they showed small or negligible effect sizes, which in turn exaggerated the effect found in our analysis. This is consistent with findings of an earlier meta-analysis, which had relaxed inclusion criteria (therefore included data from interventions not published in scientific journals), showed no sign of publication bias, and had relatively smaller effect sizes. ${ }^{29}$

Despite the number of moderators we explored in our subgroup analysis, most of those moderators were not statistically significant. We found that, in Korean children, obesity interventions had equal effect regardless of participants' sex, age, and baseline obesity status, while an earlier meta-analysis among U.S. minority children suggested that interventions limited to children who already had overweight or obesity produced greater effect sizes. ${ }^{25}$ We also found that intervention durations and the number of intervention components do not necessarily correspond with intervention effects in Korean children, while the meta-analysis among U.S. minority children found that interventions with 3-4 components were more effective than those with only $1-2 \mathrm{com}$. ponents. ${ }^{25)}$ While further studies are needed to understand why 
and how the effect of Korean interventions differs from that of U.S. interventions, clinicians must take additional precaution when making recommendations based on studies conducted outside Korea. Also, it should be noted that most of the interventions included in our analyses were intended for completion in less than a year, sometimes intended for just a couple of weeks. Considering how weight regain is common in years following obesity interventions, ${ }^{41)}$ interventions lasting longer than 1 year may show reduced effectiveness compared to interventions included in this meta-analysis.

We found that selection of intervention components may be of greatest importance when designing obesity interventions for Korean children. We found evidence that a physical activity component should be included whenever possible, such that it helps intervention children escape sedentary lifestyle. However, the inclusion of a counseling component (e.g., professional guidance) and a family support component (e.g., parent's involvement) must be done carefully, as they did not show the desired or intended effect. It is possible that interventions including counseling components were sometimes improperly implemented or failed to consider the factors that affect participants' adherence (e.g., time needed to build rapport between children and counselor). Like this study, several U.S. childhood obesity studies ${ }^{42-45)}$ reported that counseling did not play an important role in pediatric obesity. However, unlike this study, a recent meta-analysis ${ }^{46}$ that analyzed randomized controlled trials published in English from 2008 to 2018 showed that childhood obesity interventions with parental involvement produced larger effects than those without parental involvement. According to the World Health Ogarnization, ${ }^{47)}$ parents play a vital role in encouraging their children to adhere to healthy eating and physical activity which in turn is related to favorable results in obesity prevention. It is important for health educators in Korea to provide parents with health education programs that help them understand the health risks of childhood obesity. Future strategies to curb childhood obesity among Korean children should consider the vital role of parents in the prevention and treatment of childhood obesity, and rigorous evaluation of parental involvement is needed using experimental studies that focus on this topic.

Limitations of this study should be acknowledged. First, this meta-analysis compared intervention and control groups using BMI changes as the study outcome, but the 2 groups were not compared using other measures (e.g., blood pressure, fat distribution, and presence of comorbidities like diabetes) that help the clinical diagnosis of obesity. While BMI is a commonly used measure of adiposity, it does not guarantee that individuals above a certain BMI threshold for obesity are truly unhealthy and have a diagnosable form of obesity. Similarly, we do not know if interventions that effectively reduced study participants' BMI necessarily improve the health of those participants. Second, results of this meta-analysis were not corrected for age, and our subgroup analysis only compared 2 large age groups (children vs. adolescents). As intervention effectiveness may vary by intervention target's precise age, our age-related inference may be limited.
Third, inference related to "other" intervention component may be limited, as it includes methods that are not formally recog. nized as clinical intervention of pediatric obesity (e.g., acupressure). Regardless, these studies cannot be omitted, as they fit our initial inclusion criteria, and sensitivity analysis found that their omission does not significantly alter our results. Fourth, there was a sign of publication bias among included studies. We sought to minimize the effect of publication bias by using imputation; however, there is still a chance that this meta-analysis does not accurately reflect how the research findings truly are if we were to include studies that were unpublished or were published in other sources (e.g., thesis, dissertation, and commercial journals) which were not included in our search. Further investigations are needed to understand why many intervention studies on childhood obesity were unpublished in Korea. Lastly, this meta-analysis focused on quantitative synthesis, but provides no qualitative synthesis. Qualitative synthesis may provide further insight into why and how Korean obesity interventions differ from obesity interventions conducted in other countries. However, we only provided a table that summarizes study quality for assessing risk of biases. A review study with in-depth qualitative synthesis of Korean obesity interventions may be needed.

In conclusion, this study showed the effectiveness of obesity interventions for Korean children. Overall, intervention groups were strongly favored over control groups, consistent with what is suggested in the literature. Sex, age, baseline obesity status, and intervention durations did not significantly affect the BMI outcome, while interventions that include at least one physical activity component were significantly better than those that did not include any physical activity components. Despite what was suggested in the literature, we found that increasing the number of intervention components does not necessarily translate into increased intervention effects. The number of published intervention studies on childhood obesity is limited in Korea. Additional intervention studies, as well as follow-up meta-analyses, are needed to confirm findings of this meta-analysis.

\section{Footnotes}

Supplementary materials: Supplementary Figs. 1-4 can be found via https://doi.org/10.3345/cep.2021.00409.

Funding: This research was supported by the academic research fund of Namseoul University in South Korea.

Conflicts of interests: No potential conflict of interest relevant to this article was reported.

Acknowledgments: We would like to thank librarians and undergraduate research assistants at Miami University for their help with the literature search. This paper was performed with the support of the academic research fund of Namseoul University in South Korea. 
ORCID:

Siyoung Choe $@$ https://orcid.org/0000-0001-9351-1958

Jaesin Sa 1 https://orcid.org/0000-0002-2850-7239

Jean-Philippe Chaput 1 https://orcid.org/0000-0002-5607-5736

Deokjin Kim (1) https://orcid.org/0000-0003-2388-4913

\section{References}

1. Wang Y, Lim H. The global childhood obesity epidemic and the association between socio-economic status and childhood obesity. Int Rev Psychiatry 2012;24:176-88.

2. World Health Organization. Obesity and overweight [Internet]. Geneva (Switzerland): World Health Organization; 2020 [cited 2020 Apr 1]. Available from: https:/www.who.int/news-room/fact-sheets/detail/obesityand-overweight.

3. Bhurosy T, Jeewon R. Overweight and obesity epidemic in developing countries: a problem with diet, physical activity, or socioeconomic status? ScientificWorldJournal 2014;2014:964236.

4. Caleyachetty R, Echouffo-Tcheugui JB, Tait CA, Schilsky S, Forrester T, Kengne AP. Prevalence of behavioural risk factors for cardiovascular disease in adolescents in low-income and middle-income countries: an individual participant data meta-analysis. Lancet Diabetes Endocrinol 2015; 3:535-44.

5. Organisation for Economic Co-operation and Development. Obesity and the economics of prevention: fit not fat - Korea key facts [Internet]. Paris (France): Organisation for Economic Co-operation and Development; 2020 [cited 2020 Apr 1]. Available from: https://www.oecd.org/els/healthsystems/obesityandtheeconomicsofpreventionfitnotfat-koreakeyfacts.htm.

6. Ha KH, Kim DJ. Epidemiology of childhood obesity in Korea. Endocrinol Metab (Seoul) 2016;31:510-8.

7. Kim JH, Moon JS. Secular trends in pediatric overweight and obesity in Korea. J Obes Metab Syndr 2020;29:12-7.

8. Shin NY, Shin MS. Body dissatisfaction, self-esteem, and depression in obese Korean children. J Pediatr 2008;152:502-6.

9. Hong SJ, Lee MS, Lee SY, Ahn KM, Oh JW, Kim KE, et al. High body mass index and dietary pattern are associated with childhood asthma. Pediatr Pulmonol 2006;41:1118-24.

10. Kim HS, Park J, Ma Y, Ham OK. Factors influencing health-related quality of life of overweight and obese children in South Korea. J Sch Nurs 2013; 29:361-9.

11. Ham OK, Sung KM, Lee BG, Choi HW, Im EO. Transtheoretical model based exercise counseling combined with music skipping rope exercise on childhood obesity. Asian Nurs Res (Korean Soc Nurs Sci) 2016;10:11622.

12. Cote AT, Harris KC, Panagiotopoulos C, Sandor GG, Devlin AM. Childhood obesity and cardiovascular dysfunction. J Am Coll Cardiol 2013;62: 1309-19.

13. Weihe P, Spielmann J, Kielstein H, Henning-Klusmann J, WeihrauchBlüher S. Childhood obesity and cancer risk in adulthood. Curr Obes Rep 2020;9:204-12.

14. Yi DY, Kim SC, Lee JH, Lee EH, Kim JY, Kim YJ, et al. Clinical practice guideline for the diagnosis and treatment of pediatric obesity: recommendations from the Committee on Pediatric Obesity of the Korean Society of Pediatric Gastroenterology Hepatology and Nutrition. Pediatr Gastroenterol Hepatol Nutr 2019;22:1-27.

15. Strahan BE, Ahn H, Shuster J. Meta-analysis of the effects of exercise interventions on obese adolescents. J Nurs Interprof Leadersh Qual Saf 2018;2:1-9.

16. Ismail I, Keating SE, Baker MK, Johnson NA. A systematic review and meta-analysis of the effect of aerobic vs. resistance exercise training on visceral fat. Obes Rev 2012;13:68-91.

17. Peirson L, Fitzpatrick-Lewis D, Morrison K, Warren R, Usman Ali M, Raina P. Treatment of overweight and obesity in children and youth: a systematic review and meta-analysis. CMAJ Open 2015;3:E35-46.
18. Berge JM, Everts JC. Family-based interventions targeting childhood obesity: a meta-analysis. Child Obes 2011;7:110-21.

19. Kim KB, Lim KI, So WY, Park SK, Song W. A meta-analysis of the effects of exercise therapy applied in obesity studies. Korean J Obes 2007;16:17785.

20. Seo DC, Sa J. A meta-analysis of psycho-behavioral obesity interventions among US multiethnic and minority adults. Prev Med 2008;47:573-82.

21. Laviada-Molina H, Molina-Segui F, Pérez-Gaxiola G, Cuello-García C, Arjona-Villicaña R, Espinosa-Marrón A, et al. Effects of nonnutritive sweeteners on body weight and BMI in diverse clinical contexts: systematic review and meta-analysis. Obes Rev 2020;21:e13020.

22. Cotie LM, Prince SA, Elliott CG, Ziss MC, McDonnell LA, Mullen KA, et al. The effectiveness of eHealth interventions on physical activity and measures of obesity among working-age women: a systematic review and meta-analysis. Obes Rev 2018;19:1340-58.

23. Booth HP, Prevost TA, Wright AJ, Gulliford MC. Effectiveness of behavioural weight loss interventions delivered in a primary care setting: a systematic review and meta-analysis. Fam Pract 2014;31:643-53.

24. Johns DJ, Hartmann-Boyce J, Jebb SA, Aveyard P; Behavioural Weight Management Review Group. Diet or exercise interventions vs combined behavioral weight management programs: a systematic review and metaanalysis of direct comparisons. J Acad Nutr Diet 2014;114:1557-68.

25. Seo DC, Sa J. A meta-analysis of obesity interventions among U.S. minority children. J Adolesc Health 2010;46:309-23.

26. Peirson L, Fitzpatrick-Lewis D, Morrison K, Ciliska D, Kenny M, Usman Ali M, et al. Prevention of overweight and obesity in children and youth: a systematic review and meta-analysis. CMAJ Open 2015;3:E23-33.

27. Godin K, Leatherdale ST, Elton-Marshall T. A systematic review of the effectiveness of school-based obesity prevention programmes for First Nations, Inuit and Métis youth in Canada. Clin Obes 2015;5:103-15.

28. Lee HY. Effectiveness of obesity management programs: systematic review and meta-analysis. Korean J Health Educ Promot 2007;24:131-47.

29. Sung KS, Yoon YM, Kim EJ. Meta-analysis of the effects of obesity management program for children. Child Health Nurs Res 2013;19:262-9.

30. Moher D, Liberati A, Tetzlaff J, Altman DG; PRISMA Group. Preferred reporting items for systematic reviews and meta-analyses: the PRISMA statement. PLoS Med 2009;6:e1000097.

31. Kim HS, Ma YM, Park JY, Kim SH. An emotional self-regulation program for obese children: pilot study using mixed methods. Child Health Nurs Res 2013;19:187-97.

32. Lee JH, Oh S. Effects of the 26 -weeks obesity management program on obesity index, self-esteem, self-efficacy, and body image among obese elementary school children. J Korea Acad Industr Coop Soc 2016;17:83-93.

33. Yang Y, Kang B, Lee EY, Yang HK, Kim HS, Lim SY, et al. Effect of an obesity prevention program focused on motivating environments in childhood: a school-based prospective study. Int J Obes (Lond) 2017;41:1027-34.

34. Park JW, Park SH, Koo CM, Eun D, Kim KH, Lee CB, et al. Regular physical education class enhances sociality and physical fitness while reducing psychological problems in children of multicultural families. J Exerc Rehabil 2017;13:168-78.

35. Hardin AP, Hackell JM; Committee on Practice and Ambulatory Medicine. Age limit of pediatrics. Pediatrics 2017;140:e20172151.

36. Higgins JP, Altman DG, Gøtzsche PC, Jüni P, Moher D, Oxman AD, et al. The Cochrane Collaboration's tool for assessing risk of bias in randomised trials. BMJ 2011;343:d5928.

37. Cohen J. Quantitative methods in psychology: a power primer. Psychol Bull 1992;112:1155-9.

38. Higgins JP, Thompson SG, Deeks JJ, Altman DG. Measuring inconsistency in meta-analyses. BMJ 2003;327:557-60.

39. Borenstein M, Hedges LV, Higgins JP, Rothstein HR. Introduction to metaanalysis. Chichester (UK): John Wiley \& Sons, 2009.

40. Borenstein M, Hedges LV, Higgins JPT, Rothstein HR. Comprehensive meta-analysis. Version 2. Englewood (NJ): Biostat, 2006.

41. Ross R. The challenge of obesity treatment: avoiding weight regain. CMAJ 2009;180:997-8.

42. Wake M, Baur LA, Gerner B, Gibbons K, Gold L, Gunn J, et al. Outcomes and costs of primary care surveillance and intervention for overweight or 
obese children: the LEAP 2 randomised controlled trial. BMJ 2009;339: b3308.

43. Schwartz RP, Hamre R, Dietz WH, Wasserman RC, Slora EJ, Myers EF, et al. Office-based motivational interviewing to prevent childhood obesity: a feasibility study. Arch Pediatr Adolesc Med 2007;161:495-501.

44. Taveras EM, Gortmaker SL, Hohman KH, Horan CM, Kleinman KP, Mitchell K, et al. Randomized controlled trial to improve primary care to prevent and manage childhood obesity: the High Five for Kids study. Arch Pediatr Adolesc Med 2011;165:714-22.

45. McCallum Z, Wake M, Gerner B, Baur LA, Gibbons K, Gold L, et al. Outcome data from the LEAP (Live, Eat and Play) trial: a randomized controlled trial of a primary care intervention for childhood overweight/ mild obesity. Int J Obes (Lond) 2007;31:630-6.

46. Mehdizadeh A, Nematy M, Vatanparast H, Khadem-Rezaiyan M, Emadzadeh M. Impact of parent engagement in childhood obesity prevention interventions on anthropometric indices among preschool children: a systematic review. Child Obes 2020;16:3-19.

47. World Health Organization. Global strategy on diet, physical activity and health: the role of parents [Internet]. Geneva (Switzerland): World Health Organization; 2021 [cited 2021 Mar 14]. Available from: https://www. who.int/dietphysicalactivity/childhood_parents/en/.

48. Bharath LP, Choi WW, Cho JM, Skobodzinski AA, Wong A, Sweeney TE, et al. Combined resistance and aerobic exercise training reduces insulin resistance and central adiposity in adolescent girls who are obese: randomized clinical trial. Eur J Appl Physiol 2018;118:1653-60.

49. Cha HS, Park H. Effects of auricular acupressure on obesity in adolescents. Complement Ther Clin Pract 2019;35:316-22.

50. Cha HS, Park H. Effects of auricular acupressure on Korean children who are obese. J Pediatr Nurs 2020;51:e57-63.

51. Jeon JY, Han J, Kim HJ, Park MS, Seo DY, Kwak YS. The combined effects of physical exercise training and detraining on adiponectin in overweight and obese children. Integr Med Res 2013;2:145-50.

52. Kim ES, Im JA, Kim KC, Park JH, Suh SH, Kang ES, et al. Improved insulin sensitivity and adiponectin level after exercise training in obese Korean youth. Obesity (Silver Spring) 2007;15:3023-30.

53. Kim CW, Kim BT, Park KH, Kim KM, Lee DJ, Yang SW, et al. Effects of short-term chromium supplementation on insulin sensitivity and body composition in overweight children: randomized, double-blind, placebo- controlled study. J Nutr Biochem 2011;22:1030-4.

54. Kim HS, Park J, Park KY, Lee MN, Ham OK. Parent involvement intervention in developing weight management skills for both parents and overweight/obese children. Asian Nurs Res (Korean Soc Nurs Sci) 2016; 10:11-7.

55. Kim J, Kim G, Park J, Wang Y, Lim H. Effectiveness of teacher-led nutritional lessons in altering dietary habits and nutritional status in preschool children: adoption of a NASA Mission X-Based Program. Nutrients 2019; 11:1590.

56. Kim J, Kim Y, Seo YG, Park KH, Jang HB, Lee HJ, et al. Evidence-based customized nutritional intervention improves body composition and nutritional factors for highly-adherent children and adolescents with moderate to severe obesity. Nutr Res Pract 2020;14:262-75.

57. Park TG, Hong HR, Lee J, Kang HS. Lifestyle plus exercise intervention improves metabolic syndrome markers without change in adiponectin in obese girls. Ann Nutr Metab 2007;51:197-203.

58. Roh HT, Cho SY, So WY. Effects of regular taekwondo intervention on oxidative stress biomarkers and myokines in overweight and obese adolescents. Int J Environ Res Public Health 2020;17:2505.

59. Son WM, Sung KD, Bharath LP, Choi KJ, Park SY. Combined exercise training reduces blood pressure, arterial stiffness, and insulin resistance in obese prehypertensive adolescent girls. Clin Exp Hypertens 2017;39: 546-52.

60. Song JK, Stebbins CL, Kim TK, Kim HB, Kang HJ, Chai JH. Effects of 12 weeks of aerobic exercise on body composition and vascular compliance in obese boys. J Sports Med Phys Fitness 2012;52:522-9.

61. Sung KD, Pekas EJ, Scott SD, Son WM, Park SY. The effects of a 12-week jump rope exercise program on abdominal adiposity, vasoactive substances, inflammation, and vascular function in adolescent girls with prehypertension. Eur J Appl Physiol 2019;119:577-85.

How to cite this article: Choe S, Sa J, Chaput J, Kim D. Effectiveness of obesity interventions among South Korean children and adolescents and importance of the type of intervention component: a meta-analysis. Clin Exp Pediatr 2022;65:98107. https://doi.org/10.3345/cep.2021.00409 